\title{
Amelogenesis Imperfecta, hypodontia and impacted teeth - a challenging case for rehabilitation
}

IMPLANT THERAPY

OUTCOMES, PERI-IMPLANT BIOLOGY ASPECTS

\section{Jashme Patel, Helen Young, Jerry Kwok}

Guy's and St Thomas' Dental Hospital, London

\section{Abstract}

\section{Background}

Amelogenesis imperfecta (Al) is a group of hereditary disorders affecting tooth enamel structure. It affects deciduous and permanent dentition. The four main types are hypoplastic hypomineralised; hypomatured; and hypomaturation-hypoplastic with taurodontism. Presentation tends to be with sensitivity and discolouration. Clinical findings can include impacted permanent teeth, ectopic eruption, hypodontia, crown and/or root resorption, pulp calcification and rarely ankylosis. Rehabilitation begins in seeking a functional and aesthetic dentition.

\section{Aim/Hypothesis}

We present a complex case involving a patient referred from his general dental practitioner with multiple dental problems. His rehabilitation was undertaken in a secondary care (hospital) environment by various dental specialities.

\section{Material and Methods}

A 26 year old male was referred to our Joint Hypodontia clinic and assessed by both Restorative Dentistry and Oral Surgery. He had presented to his GDP and diagnosed with presented to his GDP and diagnosed with
generalised hypomineralisation, multiple generalised hypomineralisation, multiple
missing teeth, retained deciduous teeth, partially erupted molars, generalized periodontal disease and a Class III malocclusion. These clinical findings were confirmed and radiographs showed unerupted ectopic teeth, impacted teeth, ankylosis and taurodontism.

The working diagnosis of Amelogenesis Imperfecta was made and his dentition was deemed unrestorable. He was approved for dental implant funding through our institution and was listed for upper and lower dental clearance and subsequent implant placement in both arches.

Full upper and lower arch clearance was undertaken under general anaesthetic over two sessions given the technical complexity due to ankylosis and impaction. Lower arch clearance was performed first and upper arch 2 months later. The bony defects following exodontia were augmented with bovine bone substitute and haemocollagen. The sinus membrane was breached on both right and left during exodontia and repaired again with Bioss and Haemocollagen.

Results

Dental implants were placed in upper and lower arches at one sitting under general anaesthetic 4 months later. Six Astra Tech implants were placed in the upper arch in the UR5, UR3, UR 1 UL1, UL3 and UL5 sites following raising a full thickness mucoperiosteal flap. Each was torqued to $50 \mathrm{~N}$ and all but the UR2 were stable at time of closing. Cover screws were placed on all implants and primary closure obtained with 3.0 Vicryl Rapide. Five Nobel Active implants were placed in the LR6, LR3, LR1, LL3 and LL6 areas. Healing abutments were placed on all implants following stabilization again to $50 \mathrm{~N}$ torque.

Clinical review and radiographic follow up at 3 weeks post implant placement showed all implants to be stable and the soft tissues healing well.

\section{Conclusion and Clinical implications}

Amelogenesis imperfecta is a challenging condition and requires a multidisciplinary approach in order to achieve the best functional and aesthetic outcome for patients, from Paediatric dentistry in childhood to Oral Surgery and Prosthodontics in adulthood.

\section{Background and Aim}

Amelogenesis imperfecta (Al) is a group of hereditary disorders affecting tooth enamel structure. It affects deciduous and permanent dentition. The four main types are hypoplastic; hypomineralised; hypomatured and hypomaturation- hypoplastic with taurodontism.

Patients present complaining of sensitivity and discolouration of the teeth. Clinical findings include impacted permanent teeth, ectopic eruption, hypodontia, crown and/or root resorption, pulp calcification and rarely ankylosis. Rehabilitation begins in childhood and many require implant rehabilitation for a functional and aesthetic dentition.

We present a case of an adult presenting with $\mathrm{Al}$ and the discuss the complexity of the dental rehabilitation

\section{Clinical Presentation}

A 26 year old male was referred to the Joint Hypodontia clinic and assessed by Prosthodontists, Oral Surgeons and Orthodontists. Medically he was fit and well.

\section{Presentation}

- Teeth present

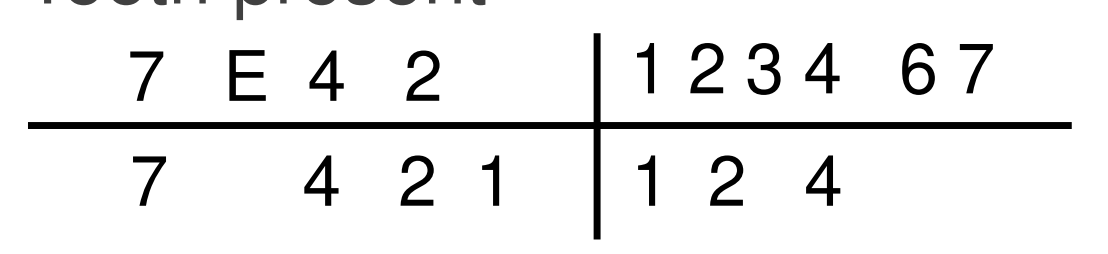

- Generalised hypomineralisation,

- Partially erupted molars,

- Generalized periodontal disease

- Class III malocclusion.

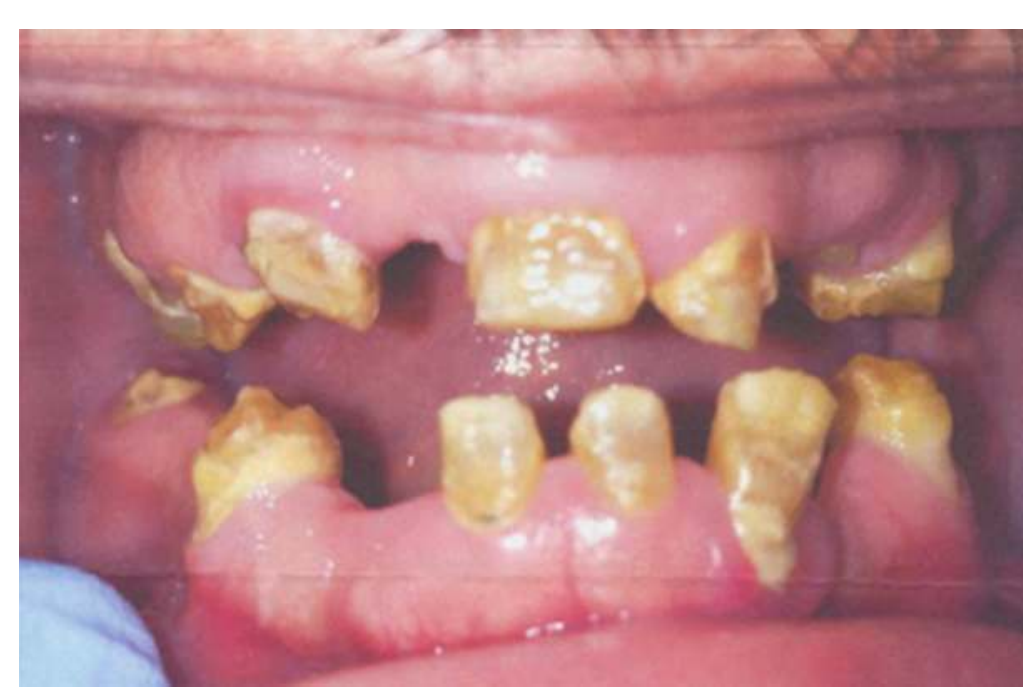

\section{Radiographs}

- Unerupted ectopic teeth

\begin{tabular}{r|rr}
85 & 5 & 8 \\
\hline 853 & 3578
\end{tabular}

- Ankylosis

- Taurodontism

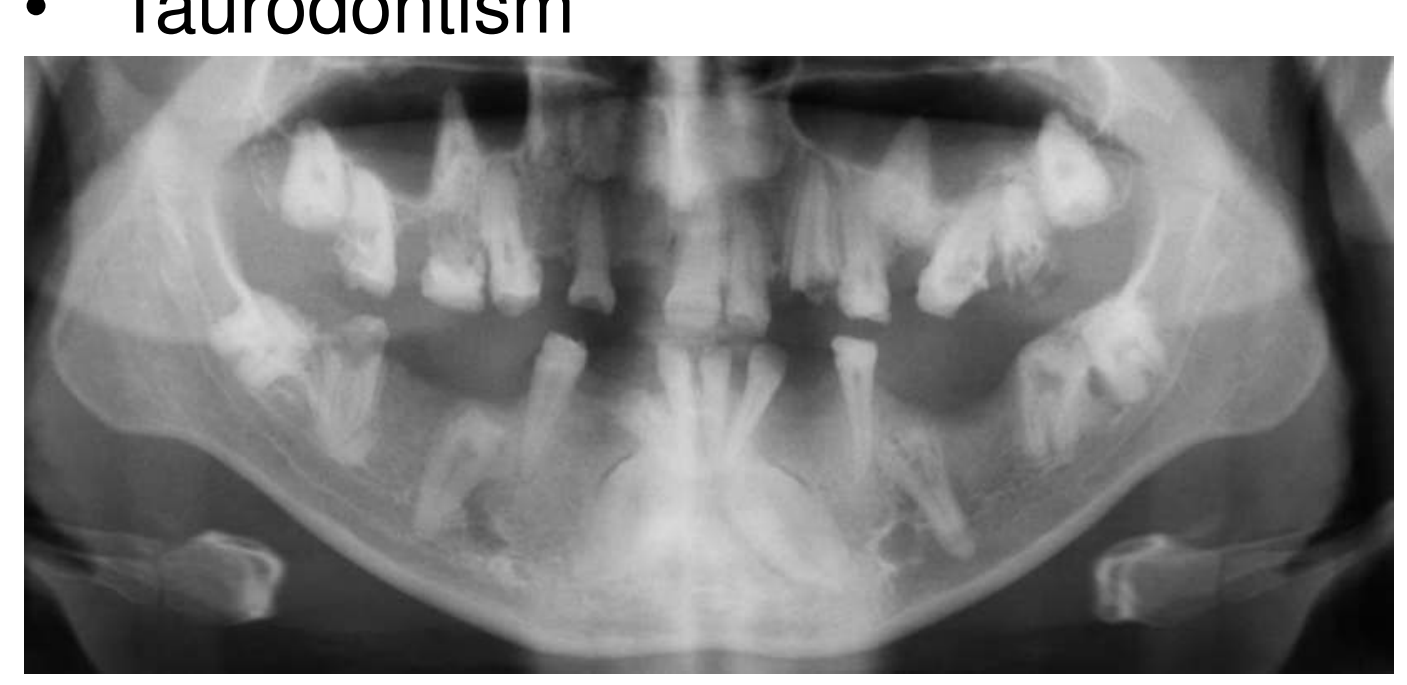

The working diagnosis of Amelogenesis Imperfecta was made and his dentition was deemed unrestorable.

\section{Treatment plan}

1. Full dental clearance

2. Implant placement

3. Implant supported over denture

\section{Extractions}

- Upper and lower arch clearance were carried out under general anaesthesia

- The extractions were over 2 sessions due technical complexity

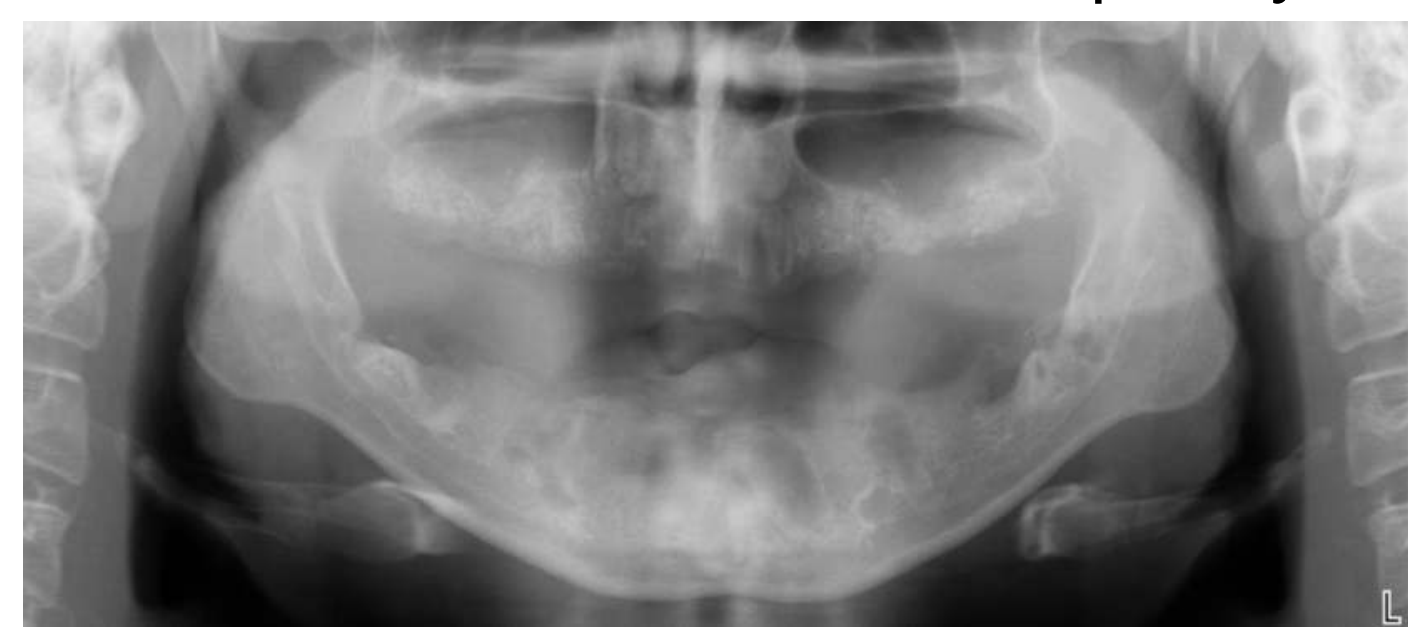

- Bony defects were augmented with bovine bone substitute \& haemocollagen

- Both maxillary sinus membranes were breached during exodontia and repaired again with Bio-Oss and haemocollagen

\section{Implant placement}

4 months later, implants were placed

- Six Astra Tech implants were placed in the UR5, UR3, UR1, UL1, UL3 and UL5 sites. Each was torqued to $50 \mathrm{~N}$ and all but the UR2 were stable at time of closing. Cover screws were placed on all implants and primary closure obtained with 3.0 Vicryl Rapide.

- Five Nobel Active implants were placed in the LR6, LR3, LR1, LL3 and LL6 areas. Healing abutments were placed on all implants following stabilization again to $50 \mathrm{~N}$ torque.

\section{Post-implant review}

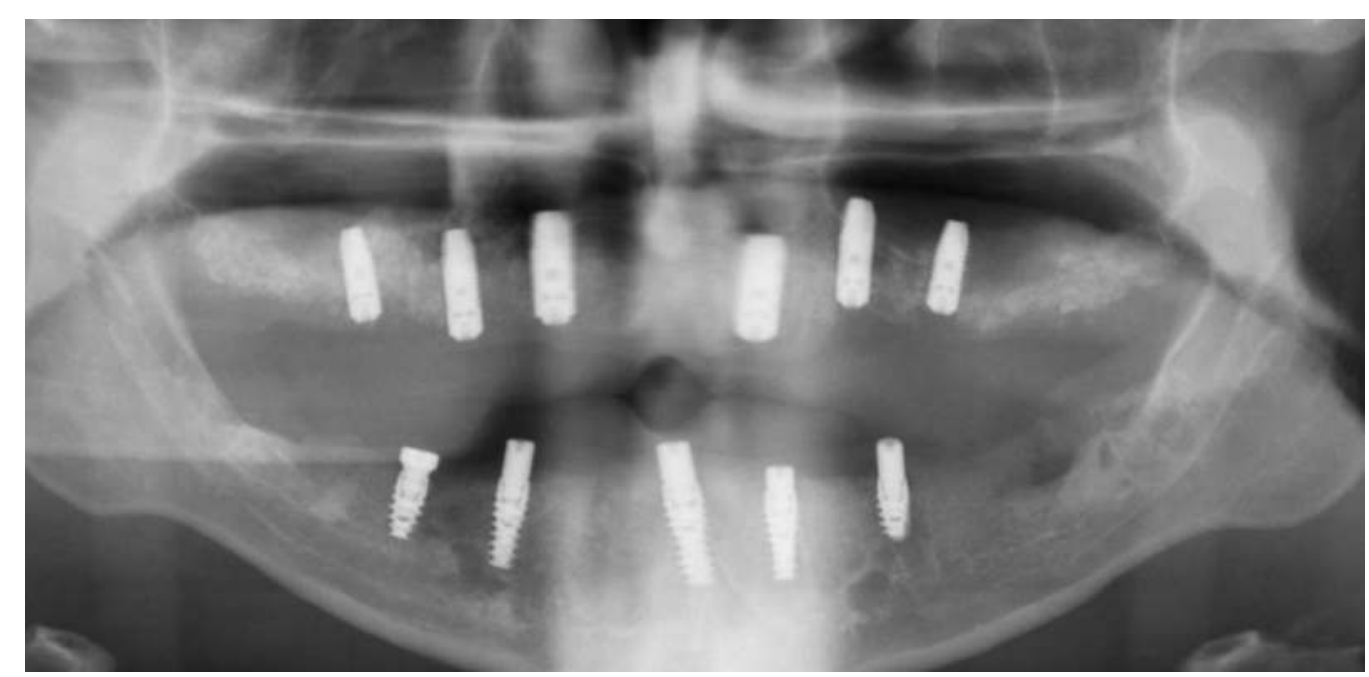

Clinical review and radiographic follow up at 3 months post implant placement showed all implants to be stable and the soft tissues healing well.

Patient is awaiting final prosthodontic treatment

\section{Conclusions}

$\mathrm{Al}$ is a challenging condition and requires a multidisciplinary approach in order to achieve the best functiona and aesthetic outcome for patients, from Paediatric dentistry in childhood to Oral Surgery and Prosthodontics in adulthood 